\title{
Creating and Using Personas in Software Development: Experiences from Practice
}

\author{
Jane Billestrup ${ }^{1}$, Jan Stage ${ }^{1}$, Anders Bruun ${ }^{1}$, Lene Nielsen ${ }^{2}$ and Kira S. Nielsen ${ }^{2}$ \\ ${ }^{1}$ Aalborg University, Department of Computer Science, 9220 Aalborg East, Denmark \\ ${ }^{2}$ IT University of Copenhagen, 2300 Copenhagen S, Denmark \\ \{jane, jans, bruun\}@cs.aau.dk, \{lene, kist\}@itu.dk
}

\begin{abstract}
Personas is a technique that supports designing and engineering interactive systems with the focus on the end-users. This paper reports from a case study, where we interviewed four software developers about their usage of personas in software development practice. The purpose of was to identify the practices of personas development in the software development industry. How the respondents perceive personas and its use does not always correlate with what is described as best practice in the literature. We found that practitioners are not using personas as stated in the literature but are developing their own practices both in regards to when and how personas are created.
\end{abstract}

Keywords: Personas, software development practice, persona creation.

\section{Introduction}

Personas is a technique that supports designing and engineering interactive systems with focus on the end-users. The common understanding of the personas technique is that a persona is a description of a fictitious person $[4,13]$ based on data collected about the target user group. The common way to represent a persona is as a text describing, and a photo depicting, the fictional user $[4,25]$. Personas has been promoted as a strong technique for providing software developers with an understanding of the prospective users of their software [5]. Personas also provides the software developers with empathy for, and engagement in, the end-users [10].

Matthews et al. [15] found that designers who had a very positive attitude towards personas were primarily those who had done extensive work with personas and had some training in the creation of personas, and used them as described by the literature. Those who had worked less with personas had a moderate or neutral opinion, and those who had not worked with personas had a negative or indifferent opinion.

This paper reports from a case study of experiences with creation and use of personas in software development practice. The case study is based on interviews with four developers who are or have been working with personas in practice. Our focus is on comparing the literature with the experiences and the perceived strengths and weaknesses of the persona technique from the perspective of the software development industry. The following section presents work related to this study. Section 3

adfa, p. 1, 2011.

(C) Springer-Verlag Berlin Heidelberg 2011 
describes the method used for data collection. Section 4 presents the results derived from the interviews. Section 5 discusses the results compared to experiences about personas reported in the literature and provides the conclusion.

\section{$2 \quad$ Related Work}

Definition of Personas. The literature originally defined personas as a text and a photo describing the character $[4,6]$. This then developed into posters, websites and handouts [14]. Personas are considered to be most useful if they are developed as whole characters, described with enough detail for designers and developers to get a feeling of its personality $[1,5,14]$. The benefits of personas are that they enable designers to envision the end-user's needs and wants, remind designers that their own needs are not necessarily the end-users' needs, and provide an effective communication tool, which facilitates better design decisions $[7,8,9,16]$.

Creating Personas. Before creating personas, a comprehensive study of the target user group is suggested. It has been suggested to acquire this information through interviews with the target user group [23] or observational studies of them [24]. Yet Chapman and Milham argue that it is not possible to verify that the created personas actually reflect the target user group [3]. It has been suggested to create 3-5 personas [26, 27], but the amount of users' one persona can represent has been questioned [3].

Personas Critique. Personas have been characterized as unreliable and not well communicated. In addition, developers lack understanding of the technique, personas are non-scientific, they are not able to describe actual people, and they prevent designers from meeting actual users $[1,11,17]$.

Personas in Practice. An inquiry of design teams in 13 Danish companies reported that personas help keep the focus on user needs instead of what the developers and designers like, and help in gaining an understanding of how the product can create value for end-users [12]. A different study has described how designers are using personas contrary to the original intended usage; instead of creating personas on research results, designers tend to base the personas on their own experiences and thoughts [2]. This will make it even harder to ensure that the right personas are created to represent the relevant user groups [3]. Problems in application of the personas technique caused by the mindset of the developers have also been reported [1, 13]. It has been suggested to overcome this by regularly sending information about the personas to the development team $[14,18]$. It seems difficult in practice to avoid making stereotypes when creating personas, and using personas does not solve the problem that Cooper originally intended to solve [28].

Combining personas and agile development, e.g. XP, has also been explored. In this case, the customer preferred a persona without a picture, merely describing a job title and maybe a name, but they do not support this as it will take away the developers' empathy for the users. Moreover, by using personas integrated in XP, the developers felt confident to make decisions without involving the onsite customer every time [21]. 


\section{$3 \quad$ Method}

We have conducted a case study about the use of personas as a development technique in four software development organizations, including if and how practitioners use personas and how they actually use this technique in practice.

Respondents. We did a survey to identify software developers who had different types of experience with using personas as part of the software development process. This involved several developers who had volunteered to participate in an interview about personas usage. From this group, we identified four different kinds of software developer experience with personas:

- Wants to start using personas as a development technique.

- Has formerly used personas as a development technique.

- Is currently using personas as a development technique.

- Has knowledge about it but never used it as a development technique.

The respondents were working as software developers or project managers. None of them had any education in user experience. All respondents had worked in the industry for at least ten years and been in their current organization for at least two years. Their organizations use an agile software development method.

Data Collection. The interviews were conducted as semi-structured qualitative interviews [19]. The interviews lasted between 22 and 55 minutes. They were recorded and then fully transcribed.

Data Analysis. All interviews were analysed using grounded theory and open coding with the Dedoose tool (https://app.dedoose.com/App/?Version=4.5.98). This resulted in seven categories that are used to structure the presentation of our findings.

\section{$4 \quad$ Findings}

This section presents the findings based on the analysis of the interviews. The findings are divided into seven sub-sections in accordance with the coding categories.

\subsection{Learning to Create Personas}

The respondents learned about the personas technique in different ways. Their first meeting with personas seems to mainly have happened by chance. Two respondents describe it this way:

R2: The first time I heard about personas was at a session with the humanities department four or five years ago. ... Microsoft has created a number of personas describing the users some years ago. They encourage us, as Microsoft consultants, to use these in our development process.

R1: I have a background as a software developer but in my former employment I worked very closely with user experience designers. 
One respondent came from a smaller company where he learned about several usability techniques and why it was important to understand and represent the users' in the development process.

\subsection{The Basis for Creating Personas}

The respondents use different ways of collecting data for the creation of personas. Yet all of them depend either on information they already have or information the customers have. None of the respondents get money or time allocated specifically to gather information about the target user group.

R1: If we don't have enough information ourselves to create the personas we will ask our customers about their usage of the existing systems.

A respondent explained that he was creating personas a bit differently than suggested by the literature. He primarily thought about the existing users and the archetypes that were standing out.

R3: We know our users quite well. Our personas are based on real users, like "can this user understand this?" We use them like personas archetypes. We do not use personas formalized. Unformalized we use personas quite a lot. Personas are based on the users who are critical towards our system; the people that make noise if they have a problem.

R2: To me a persona does not have to be too detailed in the description of the person.

None of the respondents remembered reading specific literature about personas. They had mainly learned the dos and don'ts about personas from others, or from their own experiences.

\subsection{Usefulness of Personas}

Personas are considered particularly useful when the developers are missing information about users and their work. One respondent mentioned geographical distance between designers and developers as a condition for usefulness:

R3: I find personas useful if the distance between designers and developers is substantial and they are not working side by side all day.

Another respondent explained that he found personas very useful as a substitute for onsite customers:

R1: If there is no onsite customer or employee that knows the field we are developing for very well, personas seems to be very usable. The further the designers and developers are from the users, the more value personas can bring to the development process.

This respondent's company does considerable work for the health sector, and they used to have a former nurse employed to help them understand that domain. However, this was no longer an option, so they needed to find new techniques to bring an understanding of the user groups into the development process. He thought personas could do that.

Another respondent gave an example of where he found personas to be useful; 
R2: We are creating ERP solutions. I feel that personas are a relevant tool for us. Because we are developing very specific software solutions for our customers.

This respondent also outlined different opinions about the usefulness of personas:

R2: One of my colleagues approached me one day and said the following "we live by creating solutions, not drawings." I understand his position but personally I feel that drawing up the organization first can help me understand their needs.

\subsection{Strengths of Personas}

The respondents expressed different expectations about the benefits of using personas in the development process:

R4: I believe using personas would have helped us develop a more user-friendly system.

R1: Personas can help keeping the developer's focus on the users' needs. Personas will provide the software developer with the ability to understand the users' perspective.

R2: I think that personas can provide the security for us not developing the wrong system for our user group.

One respondent added that he found personas especially useful if using a development method like the waterfall method. His argument was that when using the waterfall method the developers have only one possibility to get everything right.

R3: If using the waterfall development method you have to get everything right the first time. When developing agile it is not as critical if we make a mistake, we can change that in the next iteration as a new iteration starts every two weeks.

\subsection{Redundancy of Personas}

Two respondents stated that personas are unnecessary if user experience designers or expert users are part of the project team, so design decisions are not only left to the developers:

R4: When design is not left to the developer but is in place long before the developers begin to create the software.

R3: If you have an employee who is an expert user and knows what the user group need, personas are unnecessary.

\subsection{Weaknesses and Limitations of Personas}

The respondents agreed that using personas incorrectly can have substantial negative impact on software or product development. They also agreed that personas should not be used if there is insufficient data or if the creators are unfamiliar with personas.

R2: If the choice you make when creating the personas is wrong they will work against the design.

Another respondent raised the concern that he felt constrained by some formalized personas. Every time he was in doubt he went to look at the persona, but this meant that he got boxed in, and it stopped him from looking outside of the box. 
R3: When using personas formalized you might be a bit constrained, always going to look at the posters with the personas [...] To me it works better if I just keep them in my head. Of course our company is not that large anyway so I can just go talk to the developers if I need to change something.

Another respondent had drawn a similar conclusion:

R1: What tends to go wrong in software development is that developers tend to lock on some user requirements pretty early in the process, without documentation, and then describe the entire solution. If the user requirements or the solution change at some point, the developers tend to forget the user and their needs somewhere in the process.

Using personas requires a certain level of maturity. Another respondent's current organization was not using personas:

R1: "We are not using the personas technique at the moment. I have worked with personas in my last employment and found them very useful. I would like to introduce personas in my current employment but the company needs to be at a higher level of maturity before it would make sense. We simply have larger issues at the moment than this".

\subsection{Personas with Other Techniques}

The respondents stated that scenarios are very usable in combination with personas.

R4: Scenarios are often used in combination with personas.

R3: We have a community around our product and we host meetings with user groups, where we meet three times a year and discuss new releases and improvements.

Three respondents described that they are primarily using user stories to document the users' needs. The user stories are described by two respondents as being used instead of developing a specification of requirements.

R3: We use common sense and we are not afraid of making a mistake because it is okay if we do not get it right the first time.

\section{Discussion and Conclusion}

This paper has reported from a case study of experiences with creation and use of personas in software development practice. There are still only few studies of the actual use of personas in software development practice [20]. The purpose of this case study was to identify in detail how practitioners in the industry create and use personas in their development processes.

In many development situations, users do not know what they want, thus it is the designer's job to find out. Pruitt and Grudin [14] argue that a good design does not come from users, but from designers. This is because users do not really know what they want until they get it. It is described in the literature that personas is a useful technique to keep the developers focused on the users and their needs and give them empathy towards the personas and the end-users $[5,10]$. 
We found that the respondents perceived personas as a technique that supports designing and engineering interactive systems with a focus on the end-users. Matthews et al. [15] found that mainly developers who have been working with personas are positive in regards to a technique like personas. That was the same impression we got from our respondents. A technique like personas is also still suffering from seeming unnecessary to some developers; e.g one respondent explained that his colleague told him creating background material or drawings was a waste of time.

The practitioners do not use personas as suggested in the literature. Instead, data is collected before creating personas and it is mainly collected within their own or the customers' organization, or personas are created on the basis of real users.

Baird [22] argued that personas could be developed in a workshop while discovering requirements. One of our respondents described how they both used personas and hosted meetings with their user group regularly. These meetings were also used to get to know their users and to help get an understanding of the customers' needs.

Personas are primarily considered useful if designers and developers are not working closely together to ensure that the developers understand the intended users and use, or merely as a representation of a user if there is no onsite costumer available.

Using personas has also been described as being risky. If the personas created are targeting a wrong user group, the software solution could end up being developed for the wrong users.

Scenarios and user-stories are considered useful in combination with personas. In particular, user stories have been used to describe user situations and as a requirements specification.

The results presented in this paper are qualitative. They are based on four developers who have been interviewed in depth. The number of respondents is obviously a limitation of this study; yet only few software companies are using the personas technique in their development process, so it is very challenging to find even a few respondents with experiences from using the personas technique. It would be interesting to conduct a more extensive series of interviews practitioners about their use of personas and study how that influence the quality of the systems they develop.

\section{References}

1. Blomquist, A., Arvola, M.: Personas in action: Ethnography in an Interaction Design Team. Proc. of NordiCHI, ACM 1-1-58113-616-1/02/0010 (2002)

2. Chang, Y., Lim, Y., Stolterman, E.: Personas: From Theory to Practices. Proc. of NordiCHI. 439-442 (2008)

3. Chapman, C.N., Milham, R.: The Personas' new clothes: Methodological and practical arguments against a popular method. Proc. of HFES. 634-636 (2006)

4. Cooper, A.: The Inmates Are Running the Asylum. Indianapolis: SAMS (1999)

5. Cooper, A., Reimann, R.: About face 2.0: The essentials of interaction design. Wiley Publishing (2003)

6. Cooper, A., Reimann, R., Cronin, D.: About Face 3.0: The Essentials of Interaction Design. Wiley (2007) 
7. Long, F.: Real or Imaginary - the Effect of Using Personas in Product Design. In IES Conference, 2009 Dublin: Irish Ergonomics Review (2009)

8. Ma, J., LeRouge, C.: Introducing User Profiles and Personas into Information Systems Development. In AMCIS 2007, paper 237 (2007)

9. Miaskiewicza, T., Kozarb, K. A.: Personas and User-centered Design: How Can Personas Benefit Product Design Processes? In Design Studies 32, no. 5 (September) pp. 417-430 (2011)

10. Nielsen, L.: Engaging Personas and Narrative Scenarios. Vol 17, PhD Series. Copenhagen: Samfundslitteratur (2004)

11. Nielsen, L.: Personas - User Focused Design. Human-Computer Interaction. Springer (2012)

12. Nielsen, L., Nielsen, K. S., Stage, J., \& Billestrup, J.: Going global with Personas. In Human-Computer Interaction -INTERACT 2013 (pp. 350-357). Springer Berlin Heidelberg. (2013)

13. Pruitt, J., Adlin, T.: The Persona Lifecycle: Keeping People in Mind Throughout Product Design. Morgan Kaufmann, San Francisco (2006).

14. Pruitt, J., Grudin, J.: Personas: Practice and theory. Proc. of DUX (2003)

15. Matthews, T., Judge, T., Whittaker, S.: How do designers and user experience professionals actually perceive and use Personas? Proc. of CHI 2012. ACM.

16. Grudin, J., Pruitt, J.: Personas, Participatory Design and Product Development: An Infrastructure for Engagement. Proc. of PDC. 144-161 (2002)

17. Bak, J., Nguyen, K., Rissgaard, P., Stage, J.: Obstacles to usability evaluation in practice: a survey of software development organizations. Proc. of NordiCHI (2008)

18. Faily, S., Flechais, I.: Persona cases: A technique for grounding Personas. Proc. of CHI. 2267-2270 (2011)

19. Kvale, S.: Interview, København: Hans Reitzel (1997)

20. Billestrup, J. Stage, J. Nielsen, L. Nielsen, K.S. (2014) Persona usage in software development; Advantages and Obstacles. Proc. of ACHI p. 359-364 (2014)

21. Powell, S., Keenan, F., \& McDaid, K.: Enhancing Agile Requirements Elicitation With Personas. IADIS International Journal on Computer Science and Information Systems, 2(1), 82-95 (2007)

22. Baird, S.: Using Personas To Discover Requirements. http://philarnold.co.uk/wpcontent/uploads/2009/10/User-Personas.pdf [December 21st 2013] (2002)

23. Levin, D. (2004). Which Personas are you targeting? 5 Minute Whitepaper.

24. Quesenbery, W.: Using Personas: Bringing Users Alive. STC Usability SIG NewsletterUsability Interface. (2004)

25. Nielsen, L.: A model for Personas and scenarios creation. Roskilde, Denmark 27th November, 2003, 71. (2003)

26. Adlin, T. and Pruitt, J.: The essential persona lifecycle: Your guide to building and using personas. Morgan Kaufmann, Burlington, MA, (2010)

27. Friess, E.: Personas and decision making in the design process: an ethnographic case study. In Proceedings of CHI 2012, ACM, (2012), 1209-1218 (2012)

28. Turner, P. and Turner, S. Is stereotyping inevitable when designing with personas? Design Studies, 32, 1, 30-44, (2011) 